\title{
Rheology of magnetic alginate hydrogels
}

Cristina Gila-Vilchez, Ana B. Bonhome-Espinosa', Pavel Kuzhir ${ }^{2}$, Andrey Zubarev $^{3,4}$, Juan D.G. Duran ${ }^{1}$, and Modesto T. Lopez-Lopez ${ }^{1, *}$

${ }^{1}$ Department of Applied Physics, University of Granada, Granada, Spain

2 University Côte d'Azur, CNRS UMR 7010, Institute of Physics of Nice, ParcValrose, 06108 Nice, France

${ }^{3}$ Department of Theoretical and Mathematical Physics, Ural Federal University, Ekaterinburg, Russia

${ }^{4}$ M.N. Mikheev Instituteof Metal Physics of the Ural Branch of the Russian Academy of Sciences, Ekaterinburg, Russia

* Correspondence: modesto@ugr.es; Tel.: +34-958243206

Abstract: Magnetic hydrogels are becoming increasingly demanded for technical and biomedical applications, especially for tissue engineering purposes. Among them, alginate-based magnetic hydrogels emerge as one of the preferred formulations, due to the abundance, low cost and biocompatibility of alginate polymers. However, their relatively slow gelation kinetics provokes strong particle settling, resulting in nonhomogeneous magnetic hydrogels. Here we study magnetic hydrogels prepared by a novel two-step protocol that allows obtaining macroscopically homogeneous systems, consisting of magnetic microparticles embedded within the alginate network. We describe a comprehensive characterization (morphology, microstructure and mechanical properties under shear stresses) of the resulting magnetic hydrogels. We 
pay special attention to the effects of particle volume fraction (up to 0.33) and strength of the magnetic field on the viscoelastic properties of the magnetic hydrogels. Our results indicate that magnetic hydrogels are strongly strengthened against shear stresses as magnetic particle concentration and applied field intensity increase. Finally, we report an adaptation of the two-step protocol for the injection of the magnetic hydrogels that might be adequate for implementation in vivo. Interestingly, injected magnetic hydrogels present similar morphology and mechanical properties to noninjected hydrogels. To conclude, we report magnetic alginate hydrogels with adequate homogeneity and injectability character. These characteristics, together with the broad range of their mechanical properties, make them perfect candidates for cutting-edge technology.

\section{Introduction}

Hydrogels are cross-linked networks of hydrophilic polymer chains dispersed in a continuous aqueous medium [1]. Due to their soft consistency and flexibility, their high water content, and the versatility of their mechanical properties, hydrogels have found diverse applications in technology and biomedicine [1-8]. Current basic research within this field largely focus on stimuli-responsive hydrogels, characterized by changes of their properties in response to a stimulus, such as temperature, chemicals or $\mathrm{pH}$ [9]. This smart behavior allows applications of hydrogels for the detection of analytes or as reservoirs for the controlled release of a drug [10-12].

Magnetic gels or ferrogels constitute one of the most important categories among stimuli-responsive gels [13-14]. They consist of suspensions of magnetic particles embedded within a polymer network swollen by liquid solutions [15-17]. Because of 
their composition, magnetic gels combine in a single material the paramagnetic behavior provided by the particles and the softness of the polymer network. As a consequence they possess the unique feature among soft matter of responding to applied magnetic fields, a characteristic that can be used for example for inducing shape changes, modifing the mechanical properties, or provoking the controlled release of absorbed drugs or cells [18-21].

The specific properties of any given magnetic gel depend mainly on the polymer network and the embedded magnetic particles. The rigidity of hydrogels is mainly predefined by the polymer network, with networks built by chemical bonding being rather rigid, whereas physical networks (built by ionic bonding, $\mathrm{H}$-bonding) tend to be more flexible and even injectable [22]. The size of the particles also plays a relevant role, with small (nanosized) particles only experiencing a weak attraction between themselves under moderate magnetic fields, whereas large (micronsized) particles interact strongly even at low magnetic fields [23]. Accordingly, strong magnetic fieldinduced changes of the elasticity of magnetic hydrogels consisting of micronsized magnetic particles have been previously reported -see for example Ref. [24].

Among polymers used for the preparation of magnetic hydrogels, alginate stands as the preferred choice for many researchers. This is motivated by the low cost and biocompatibility of alginate salts, together with the ease of preparation of ionic alginate hydrogels [25]. However, the preparation of magnetic field-responsive alginate hydrogels with homogeneous cross-linking density and homogeneous distribution of the magnetic particles within the polymer network is an open field of research. Furthermore, within the same context, the preparation of injectable magnetic hydrogels 
is of the greatest relevance, since injectability is one of the main requirements for minimally invasive procedures, particularly in tissue engineering and drug delivery applications.

Bearing this in mind, we designed a novel two-step protocol for the preparation of homogeneous magnetic hydrogels consisting of micronsized iron particles embedded within an alginate polymer network. Our protocol allows obtaining homogeneous hydrogels containing as much as 0.33 volume fraction of magnetic particles, that demonstrated strong magnetic field-responsive behavior. We report this protocol in this paper and present a comprehensive characterization of the morphology, microstructure and mechanical properties of the resulting magnetic hydrogels. Finally, we describe an adaptation of the protocol for the injection of the magnetic hydrogels that might be suitable for implementation in vivo.

\section{Materials and Methods}

\subsection{Preparation of the hydrogels and magnetic hydrogels}

The simplest approach for the preparation of magnetic alginate hydrogels is the dispersion of magnetic particles in a solution of sodium alginate, followed by the addition of a source of calcium ions $\left(\mathrm{Ca}^{2+}\right)$. Each calcium ion bonds by ionic interaction to two negatively charged alginate chains (valence -1), giving rise to the formation of an ionic polymer network [25]. When the source of calcium ions is a highly soluble salt, such as $\mathrm{CaCl}_{2}$, the resulting hydrogels lack homogeneity due to inhomogeneous crosslinking density, which is appreciably higher close to the place of addition of the calcium source [26]. This inhomogeneity can be prevented by using poorly soluble salts (e.g. $\left.\mathrm{CaCO}_{3}\right)$ as source of calcium ions, which gives rise to a slow gelation kinetics that 
results in homogeneous cross-linking density [27]. However, a slow gelation kinetics should be avoided in the case of magnetic hydrogels based on micronsized magnetic particles -note that these particles possess a high mass density and negligible Brownian motion that provoke particle settling in water-based solutions and, therefore, undesired strong particle gradients, and even phase separation, in the resulting hydrogels if gelation kinetics is slow. In order to simultaneously solve the lack of homogeneity in the density of cross-linking (if highly soluble calcium salt is used) and the strong particle gradients (if poorly soluble calcium salt is used) we designed a novel two-step protocol that, by contrast to one-step protocols, allows obtaining magnetic hydrogels with homogeneous distribution of particles and cross-linking density.

For this aim, we followed a standard sample preparation procedure in order to ensure reproducibility of the results. First of all, we prepared a polymer network using sodium alginate (empirical formula $\left.\left(\mathrm{C}_{6} \mathrm{H}_{7} \mathrm{NaO}_{6}\right)_{\mathrm{n}}\right)$ obtained from the extracellular matrix of brown algae, with a molecular weight of $176.10 \mathrm{~g} / \mathrm{mol}$ (Sigma Aldrich, USA). The sodium alginate was dissolved in distilled water at a concentration of $1 \% \mathrm{w} / \mathrm{v}$. Then, calcium carbonate $\left(\mathrm{CaCO}_{3}\right)$ was used in combination with D-glucono- $\delta$-lactone (GDL) (Sigma Aldrich, USA) as a source of calcium ions to initiate gelation -note that GDL hydrolyses in water to gluconic acid, which enhances the solubility of $\mathrm{CaCO}_{3}$ as a consequence of the resulting acidification of the medium. For a final volume of $5 \mathrm{~mL}$ of the sodium alginate solution, we added $7.5 \mathrm{mg}$ of $\mathrm{CaCO}_{3}$ and $26.7 \mathrm{mg}$ of GDL, and we stirred the mixture by a vortex mixer until it was macroscopically homogeneous. Then, we placed the mixture in a Petri dish and we left it at rest at room temperature in a water-saturated atmosphere for gelation. In order to get a magnetic hydrogel, we broke the gelling mixture by a vortex mixer $90 \mathrm{~min}$ after the gelation was initiated. After 
breaking the hydrogel, we added the magnetic phase and we sonicated the resulting mixture for 10 min before placing it at rest again in a Petri dish. As magnetic phase (iron particles) we used Fe-CC powder and Fe-HQ powder, both supplied by BASF (Germany) -note that unless specified otherwise, Fe-CC powder was used for the experiments shown in this manuscript. The thin silica coating of the Fe-CC powder is the main difference between both powders. These powders consisted of spherical micronsized particles of diameter $1.4 \pm 0.6 \mu \mathrm{m}$ (Fe-CC) and $0.9 \pm 0.3 \mu \mathrm{m}$ (Fe-HQ), as obtained by electron microscopy images, and had volumetric mass densities of $7.71 \pm$ $0.19 \mathrm{~g} \cdot \mathrm{cm}^{-3}(\mathrm{Fe}-\mathrm{CC})$ and $7.88 \pm 0.16 \mathrm{~g} \cdot \mathrm{cm}^{-3}(\mathrm{Fe}-\mathrm{HQ})$, as measured by a pycnometer. Both powders presented a typical paramagnetic behavior with saturation magnetization $M_{S}=1587 \pm 2 \mathrm{kAm}^{-1}$ for Fe-CC and $M_{S}=1721 \pm 2 k A m^{-1}$ for Fe-HQ, as measured by SQUID magnetometry. Finally, we added $5 \mathrm{~mL}$ of calcium chloride $\left(\mathrm{CaCl}_{2}\right)$ (Sigma Aldrich, USA) at a concentration of $45 \mathrm{mM}$ to the magnetic sample previously placed in a Petri dish and we kept it overnight at a room temperature in a water-saturated atmosphere. For comparison, we prepared nonmagnetic hydrogels following the same protocol without particle addition.

\subsection{Macroscopic appearance, swelling and microscopic structure of hydrogels}

We analized the macroscopic appearance of the hydrogels by direct observation and optical photography with a digital camera. Swelling tests were carried out in order to obtain information about the cross-linking density and porous structure of the hydrogels. For this aim, we proceeded as it follows. First of all, we placed the hydrogels in dry Petri dishes and we measured their mass by means of a digital microbalance. Then, we dried the hydrogels for 24 hours at room temperature in contact with blotting 
paper in order to obtain their mass after dehydration. Finally, we rehydrated the hydrogels again by submerging them in water for some additional 24 hours and

obtained their mass. The microscopic structure of the hydrogels was analized by ESEM (Enviromental Scanning Electron Microscopy) images, which were perfomed using a FEI Quanta 400 ESEM equipped with a Peltier effect cooling stage.

\subsection{Rheological characterization of the hydrogels under shear}

We determided the rheological properties under shear of both magnetic and nonmagnetic hydrogels by using a rotational (magneto)rheometer (Physica MCR 300) with a plate-plate geometry of $20 \mathrm{~mm}$ of diameter and at a constant temperature of $25 \pm 0.1$ ${ }^{\circ} \mathrm{C}$. For this aim, we placed the disk-like samples obtained at the end of the two-step protocol in the measuring system of the rheometer, with the bottom surface of the samples in contact with the lower plate of the measuring system. In some specific experiments, and in order to investigate the potential influence of the existence of a vertical gradient in concentration of magnetic particles within the hydrogel, we turned up-and-down the samples prior to their placement in the mesuring system of the rheometer -i.e., in these cases, the top surface of the disk-like hydrogels (as viewed with reference to the direction of gravity during cross-linking) was placed in contact with the lower plate of the measuring system.

First we determined the linear viscoelastic region (LVR) of the different hydrogels, by subjecting them to deformation amplitude sweep tests at a constant frequency of 1 $\mathrm{Hz}$ and stepwise increasing shear strain amplitude, $\gamma_{0}$. From these measurements we obtained the values of the storage $\left(G^{\prime}\right)$ and loss $\left(G^{\prime \prime}\right)$ moduli as a function of $\gamma_{0}$. From the resulting curves we calculated the characteristic $G^{\prime}$ and $G$ '’ values within the LVR 
by averaging for the total extension of the LVR. Afterwards, we performed frequency sweep tests at a fixed shear strain amplitude $\left(\gamma_{0}=0.03 \%\right)$ within the LVR, and increasing frequency in the range 0.15 to $15 \mathrm{~Hz}$. From these measurements we obtained the values of $G^{\prime}$ and $G^{\prime \prime}$ as a function of frequency. Both amplitude and frequency sweeps were carried out under the application of magnetic fields of different intensity within the range $0-282 \mathrm{kA} / \mathrm{m}$.

Finally, we subjected the samples to magnetic field sweep tests. For this aim, we subjected the samples to an oscillatory strain of fixed amplitude $\left(\gamma_{0}=0.03 \%\right)$ and frequency $(1 \mathrm{~Hz})$ and increased stepwise the intensity of the applied magnetic field from 0 to $282 \mathrm{kA} / \mathrm{m}$. For the total duration of the experiment (300 s) we monitored the values of the viscoelastic moduli as a function of time.

We imposed a constant compressive normal force of $0.1 \mathrm{~N}$ during measurements to ensure that there was always contact between the upper plate of the rheometer and the hydrogels -note that magnetic hydrogels experience magnetostriction, which for a fixed gap might result in loss of contact between the upper plate and the sample. Differences in the gap thickness within an amplitude or frequency sweep were always smaller than 10\%. Furthermore, we created a water-saturated atmosphere around the sample to avoid solvent loss, and used a fresh sample for each experiment to discard the influence of changes in the microstructure.

\subsection{Injectability study. Protocol and characterization after injection}

Finally, we analyzed the potential injectability of the magnetic hydrogel. For this aim, we chose magnetic hydrogels containing 0.046 volume fraction of magnetic 
particles and subjected them to an injection protocol with potential to be implemented in in vivo applications. This protocol consisted in the following steps.

i. Magnetic alginate hydrogels were prepared inside syringes by following the protocol described above in the subsection 2.1, except for the final addition of the calcium chloride solution. A stock of these magnetic hydrogels might be stored for several days until their use was required. For the present study, we stored them overnight in a water-saturated atmosphere at room temperature.

ii. Prior to injection, we partially dehydrated the hydrogels until they lost about $20 \%$ of their total mass. For this aim, we extracted them from the syringes (by their rear opening) and placed them in Petri dishes inside an oven at $35{ }^{\circ} \mathrm{C}$ for two hours. The reason for this partial dehydration was to gain some space for the extra solution that was required to be added after injection. Otherwise, if we did not perform this partial dehydration, some supernatant solutions (not absorbed in the hydrogels) remained, something undesired from the viewpoint of in vivo applications.

iii. Afterwards, we placed the hydrogels back inside syringes (note that the hydrogels maintained their shape during partial dehydration) and injected them where desired.

iv. At some given time (ranging from 0 to 120 minutes) after their injection, we added by injection a total volume equal to the volume of water lost during the partial dehydration (step ii) of a solution of $\mathrm{CaCl}_{2}$. The concentration of $\mathrm{CaCl}_{2}$ in this solution was adjusted so that the final molarity of $\mathrm{CaCl}_{2}$ in the hydrogel was $22.5 \mathrm{mM}$-remark that this is the same molarity as in the protocol described in subsection 2.1. 
We evaluated by a simple laboratory study the feasibility of injection of the magnetic hydrogel in a macroporous structure, which represented an approximate model of a biological tissue. For this aim we used a commercial polyurethane foam (Procusur, Spain) having an approximate compressive modulus of $4600 \pm 100 \mathrm{~Pa}$. We drilled a cup-like hole of approximately $1 \mathrm{~cm}$ in diameter and $2 \mathrm{~cm}$ in depth in the foam. Then, we covered the surface of the foam having the hole with an additional foam of the same size, and maintained them in contact for the total length of the experiments. Afterwards we followed the protocol described above for the injection of the magnetic hydrogel in the drilled space between the two foams. At the end of the experiment we separated the foams and recovered the magnetic hydrogel from the hole.

In all cases, we recovered the resulting magnetic hydrogels after 24 hours of the addition of the $\mathrm{CaCl}_{2}$ solution. Then, we analyzed their macroscopic integrity by direct observation and optical microscopy with a digital camera. We also characterized the viscoelastic properties of the injected hydrogels. For this aim, we followed the protocol described above for the injection of the magnetic hydrogel in a Petri dish, in order to obtain a dish-like hydrogel, following afterwards the same rheological protocols described above in subsection 2.3 for the characterization.

\subsection{Statistics}

For each set of experimental conditions we measured at least 3 different samples. In the case of rheological measurements we performed 3 different repetitions for each sample. Thus, in total we have at least 3 values in swelling experiments and 9 in rheological measurements for each set of experimental conditions. An exception to this were magnetic sweep tests for which we did not perform repetitions. In this manuscript 
we provide the corresponding mean values and standard deviations of performed experiments.

\section{Results and discussion}

\subsection{Macroscopic appearance, swelling and microscopic structure of hydrogels}

Both nonmagnetic and magnetic hydrogels retained the shape of the disk-like container used for their preparation (Figure 1). Nonmagnetic hydrogels were transparent and presented a homogeneous macroscopic appearance. Magnetic hydrogels were black in color and also presented a homogeneous macroscopic structure, even for a volume fraction of magnetic particles $(\Phi)$ as high as 0.33 . Note that the hydrogels maintained their shape and integrity under the manipulation and measurements performed in this work. An exception for this was magnetostriction of the magnetic hydrogels under high enough intensity of applied magnetic field (Table 1). Besides magnetostriction, for 0.09 particle volume fraction or higher, the hydrogels suffered irreversible macroscopic breakage when subjected to shear strains out of the LVR under applied magnetic field (Figure 1c).

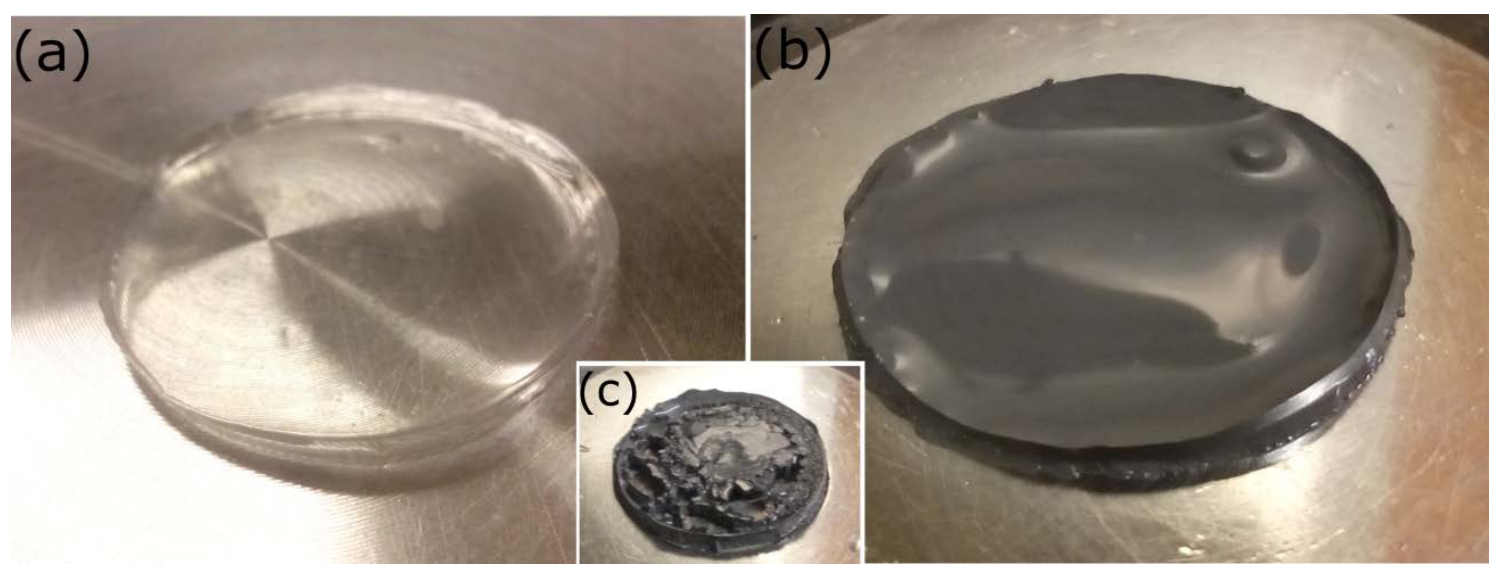


Figure 1. (a) Nonmagnetic hydrogel; (b) Magnetic hydrogel containing 0.29 volume fraction of magnetic particles -magnetic hydrogels containing different concentrations of particles presented a similar aspect; (c) Magnetic hydrogel containing 0.17 volume fraction of magnetic particles after being subjected, under the presence of an applied magnetic field of $282 \mathrm{kA} / \mathrm{m}$, to an amplitude sweep test with maximum amplitude of shear strain, $\gamma_{0-\max }=100 \%$.

Table 1.Maximum change in height experienced by the magnetic hydrogels under the maximum applied magnetic field under study $(282 \mathrm{kA} / \mathrm{m})$.

\begin{tabular}{ccccccc}
\hline $\begin{array}{c}\text { Fe-CC volume } \\
\text { fraction }\end{array}$ & 0.046 & 0.09 & 0.17 & 0.23 & 0.29 & 0.33 \\
\hline $\begin{array}{c}\text { Approx. height } \\
\text { increment }\end{array}$ & $<10 \%$ & $<10 \%$ & $50 \%$ & $50 \%$ & $50 \%$ & $70 \%$ \\
(magnetostriction) & & & & & & \\
\hline
\end{tabular}

We also analyzed the swelling behavior of the hydrogels (Table 2, Figure 2). Fully hydrated hydrogels presented strong differences in masses due to the mass of the embedded particles, the amount of absorbed water being similar in all cases. The amount of absorbed water is directly related to the porosity of the hydrogels and thus we can conclude that the porosity of the hydrogels was not affected by the presence or content of the magnetic particles. Note that the porosity of hydrogels plays an important role in biomedical applications. For example, when hydrogels are used as scaffolds for tissue regeneration, a large porosity and porous size is 
required for the diffusion of cells, nutrients and oxygen, as well as for the removal of waste.

Table 2. Experimental data corresponding to the swelling characterization of the hydrogels. $W_{\mathrm{i}}$ is the initial mass of the hydrogel, $W_{\mathrm{d}}$ is the hydrogel mass after dehydration, and $W_{\mathrm{r}}$ the mass after rehydration.

\begin{tabular}{cccccc}
\hline $\begin{array}{c}\text { Fe-CC } \\
\text { volume }\end{array}$ & $W_{i}(\mathbf{m g})$ & $W_{d}(\mathbf{m g})$ & $W_{d} / W_{i}$ & & $W_{r}(\mathbf{m g})$ \\
fraction & & & $(\%)$ & & $(\%)$ \\
\hline 0 & $440 \pm 70$ & $7.6 \pm 0.5$ & $1.7 \pm 0.3$ & $84.9 \pm 0.3$ & $19 \pm 3$ \\
0.046 & $399 \pm 19$ & $160 \pm 10$ & $40 \pm 3$ & $269 \pm 17$ & $67 \pm 5$ \\
0.09 & $700 \pm 70$ & $330 \pm 30$ & $47 \pm 6$ & $440 \pm 40$ & $63 \pm 8$ \\
\hline
\end{tabular}

For all hydrogels there was considerable loss of mass when they were dehydrated. The mass loss was about $98 \%$ of the initial mass in the case of nonmagnetic hydrogels and about $50 \%$ of their mass for a magnetic hydrogel containing 0.09 volume fraction of magnetic particles. These results make sense taking into account that, apart from magnetic particles, water was the main compound of hydrogels in terms of mass. After dehydration, we tried to rehydrate hydrogels by immersing them in water. However, for both nonmagnetic and magnetic hydrogels there only was a marginal rehydration, with most of the increase in mass due to water adsorption (superficial) on the hydrogels instead of the desired water absorption (bulk) -see some examples in Figure 2b and 2d. Note also that the rehydration was similar for the different hydrogels in terms of the mass 
recovered. This reduced capacity of rehydration of alginate hydrogels is an indication of the formation of stable hydrogen bonding among polymer chains by drying [28].
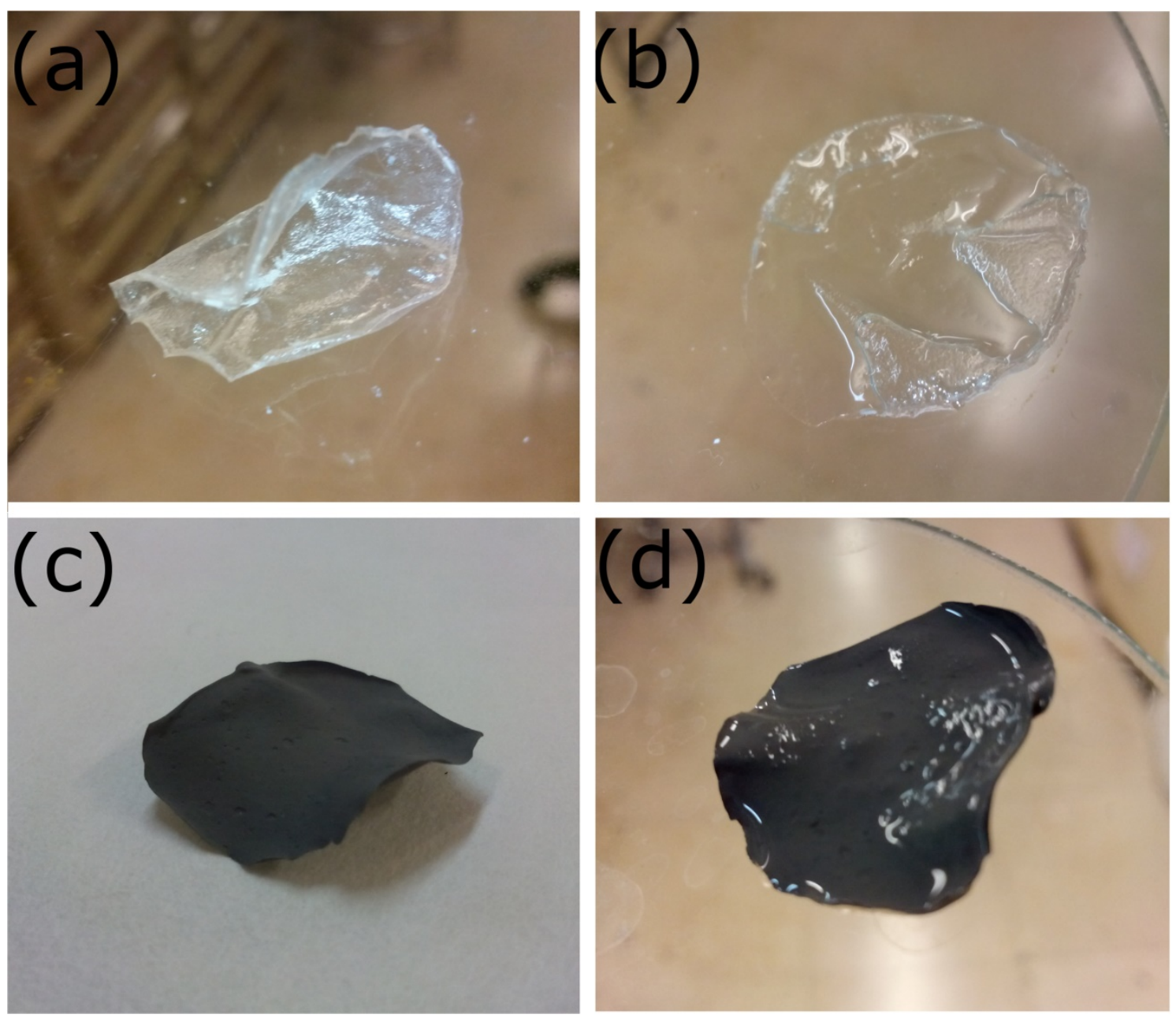

Figure 2. Nonmagnetic hydrogel (a) after the dehydration process and (b) after the rehydration process. Magnetic hydrogel containing 0.046 volume fraction of magnetic particles (c) after the dehydration process and (d) after the rehydration process -other magnetic hydrogels presented a similar aspect.

With respect to the microstructure, nonmagnetic alginate hydrogels presented a dense homogeneous web-like microstructure (Figure 3a). On the other hand, 
magnetic hydrogels presented a web-like microstructure disrupted by the presence of the magnetic particles (Figures 3b-d). As observed, the particles seem to be mainly encapsulated within the alginate polymer network (Figure 3c), although linkage between the polymer strands and the surface of the particles seems to take place (see Figure 3d). Note finally that due to the much larger size of the particles in comparison with the pore size of the polymer network, the latter can be considered as a continuous medium with respect to iron microparticles (Figure 3b).
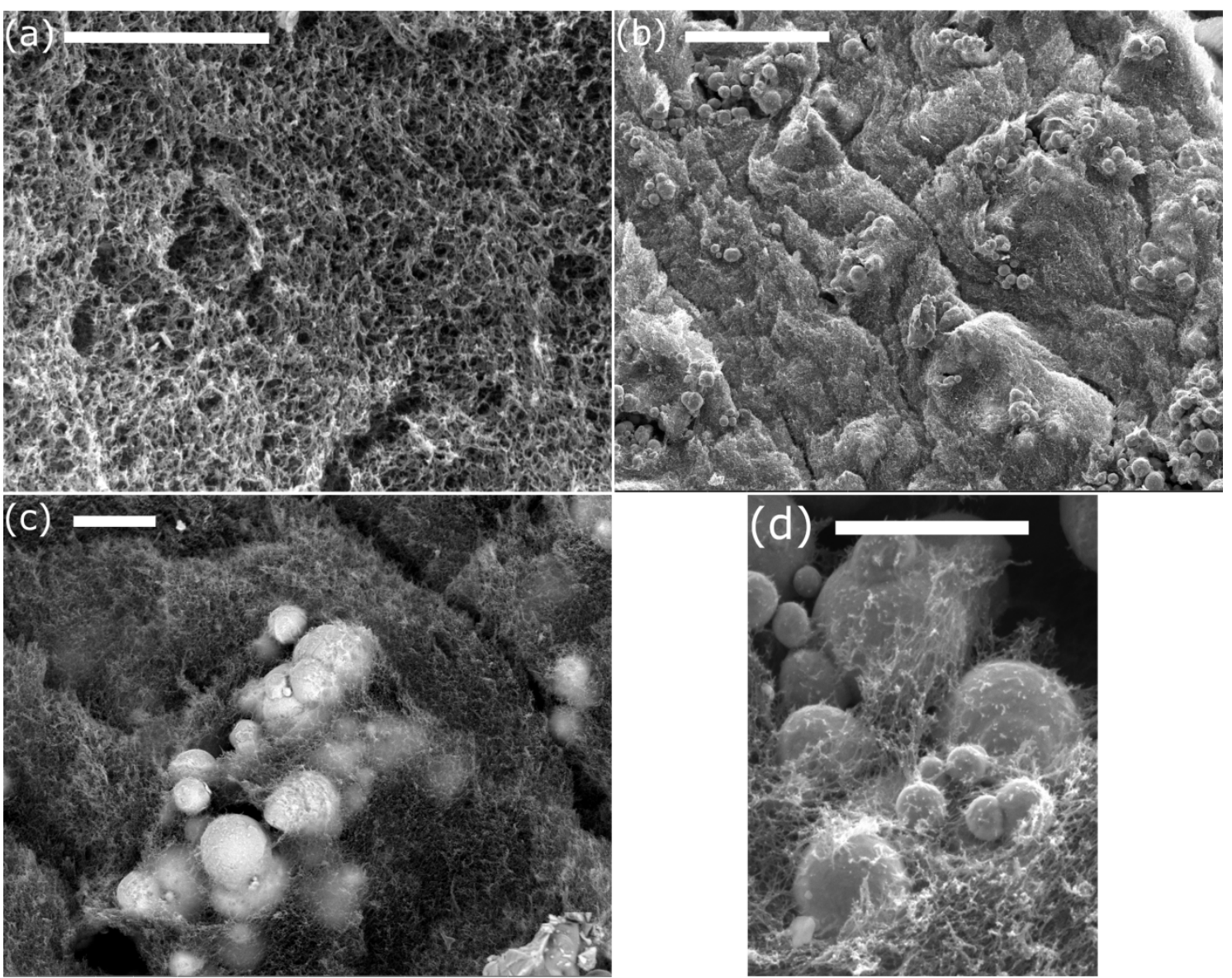

Figure 3. Scanning electron microscopy images of hydrogels. (a) Nonmagnetic hydrogel; (b), (c) and (d) magnetic hydrogels containing 0.01 volume fraction of Fe-CC particles. Note the presence of some polymer strands linked to the 
surface of the particles, more evident in part (d). Scale bar length: (a), (c), (d) 4 $\mu \mathrm{m}$; and (b) $30 \mu \mathrm{m}$.

\subsection{Rheological characterization of the hydrogels under shear}

\subsubsection{Rheological characterization in the absence of an applied magnetic field}

Firstly we analyzed the dependence of the storage ( $\left.G^{\prime}\right)$ and loss ( $\left.G^{\prime \prime}\right)$ moduli as a function of the strain amplitude in oscillatory measurements of fixed frequency of $1 \mathrm{~Hz}$. For both nonmagnetic and magnetic hydrogels the experimental curves showed a typical trend of a viscoelastic solid-like material, characterized by $G^{\prime}$ values much larger than G', values at low strain amplitude (Figure 4). As observed, both viscoelastic moduli presented an initial plateau-like region, which is identified with the LVR. Then, as the strain amplitude increased, $G$ ' experimented a sharp decreased, which was accompanied by an initial increase of $G$ ', up to a maximum (peak value), followed by a sharp decrease at higher values of the strain amplitude. This region where the plateau values of $G^{\prime}$ and $G^{\prime \prime}$ are no longer maintained is known as nonlinear viscoelastic region. Within this region, the internal structure of the hydrogels suffered from irreversible deformation and breakage, which provoked the observed decrease of elasticity (evidenced by the decrease of $G^{\prime}$ ). Concerning the trend for $G^{\prime}$,' the peak value corresponded to a yielding point, at which the dissipation of energy (and thus the irreversible destruction of the gel) is maximum [29]. The relevance of the G'” peak, in comparison with the neighboring values, decreases strongly with the concentration of magnetic particles. In fact, the gels containing a volume fraction higher than 0.30 did not show any peak (values not shown here for brevity). This can be taken as an evidence 
of the connection of the peak with the flexible polymeric structure of the gel -very likely the peak reflects destruction of the polymer structure, which becomes relatively less important as the concentration of magnetic particles increases. Concerning the value of the strain at which the peak took place, it decreased abruptly when particles were included in the formulation with respect to the nonmagnetic gel. Then, this magnitude showed a trend to increase slightly with concentration of magnetic particles (Figure 5a).
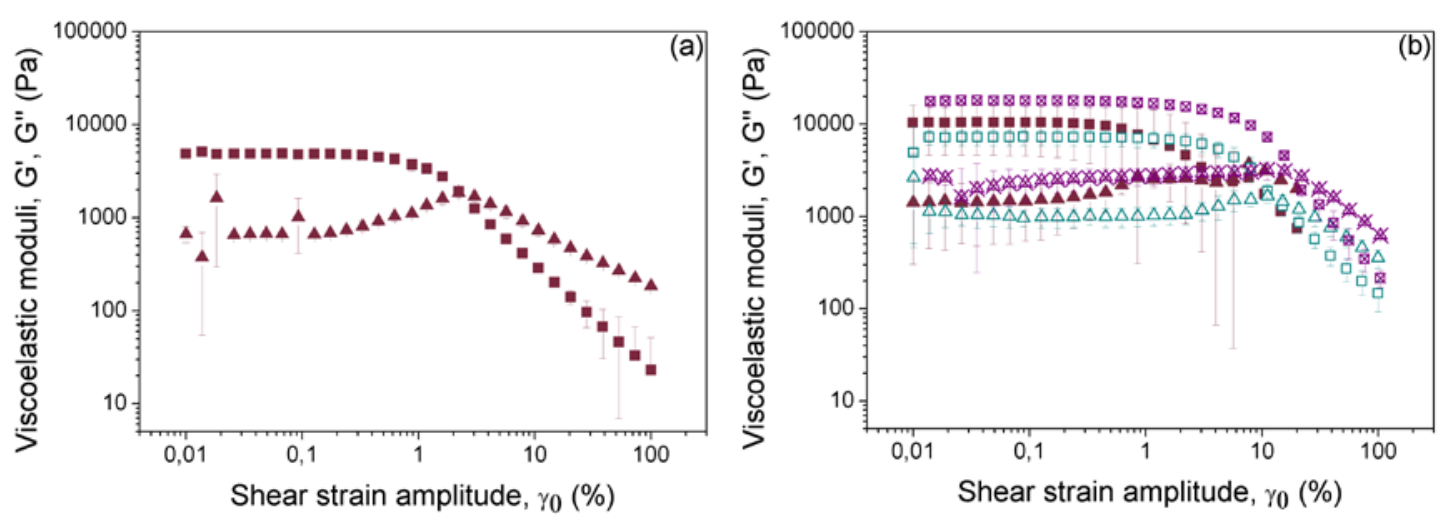

Figure 4. Storage ( $\boldsymbol{\square})$ and loss ( $\boldsymbol{\Delta})$ moduli as a function of strain amplitude for oscillatory measurements at a frequency of $1 \mathrm{~Hz}$. (a) Nonmagnetic hydrogel; (b) magnetic hydrogel containing 0.046 volume fraction of magnetic particles. The comparison between measurements for magnetic hydrogels containing FeCC particles placed on the rheometer plate without turning (solid symbols) and after turning up-and-down (open symbols) is included in (b). Results for magnetic hydrogels containing Fe-HQ particles (crossed symbols) are also included for comparison in (b). Note that the same axis scales are used in both parts. 
As evidenced from curves of Figure 4, the inclusion of magnetic particles within the composition of the hydrogels resulted in an enhancement of the viscoelastic moduli (similar results were obtained for other concentrations of particles, not shown here). In order to better analyze this effect, we plotted the average values of $G^{\prime}$ and $G$ '” corresponding to the LVR as a function of the concentration of magnetic particles (Figure 5b). At this point it is important to note that different criteria can be found in the literature to define the extension of the LVR [30]. In this work we used the criteria of [31], which defined the limit of the LVR as the point where the storage modulus deviates $10 \%$ from the plateau value. The value of this limit as a function of the volume fraction of magnetic particles showed a sharp decrease around $0.05-0.10$ and almost flat trends above and below this concentration range -data not shown here for simplicity. Concerning the trends of $G^{\prime}$ and $G^{\prime}$ ', as observed there is an increase of both magnitudes with particle concentration (Figure 5b). The effect of solid inclusions on mechanical properties of composite materials is a subject studied from the theoretical and experimental viewpoints [32]. In particular, it has been shown that due to full equivalence between equations of motion of the incompressible elastic solid and of the incompressible viscous fluid, the concentration dependence of the shear moduli of the former has exactly the same form as the concentration dependence of the shear viscosity of the latter under restriction of the same spatial and orientational distribution of particles in both media. In the case of perfectly rigid spherical inclusions, at low concentration of the disperse phase, the classical Einstein's formula gives a good prediction. As the concentration of the disperse phase is increased Batchelor's formula [33] first (up to volume fraction of 0.09) and Krieger-Dougherty (KD) equation [34] for even higher concentration become adequate expressions for the prediction of the change 
in the storage modulus of the composite material with particle content. Under hypothesis of incompressible hydrogel, which is expected to hold since the hydrogel is mostly composed of water (incompressible liquid) and of rigid metallic particles, prediction of $\mathrm{KD}$ equation for the storage modulus of a continuous medium with rigid spherical inclusions reads as it follows [34]:

$$
G^{\prime}=G^{\prime}{ }_{0}\left(1-\Phi / \Phi_{m}\right)^{-[\eta] \Phi_{m}} ; \quad G^{\prime \prime}=G^{\prime \prime}{ }_{0}\left(1-\Phi / \Phi_{m}\right)^{-[\eta] \Phi_{m}}
$$

Here $\Phi$ is volume fraction of the inclusions (individual microparticles or their aggregates), $\Phi_{m}$ is their maximum-packing volume fraction, $G_{0}^{\prime}, G^{\prime \prime}{ }_{0}$ are respectively the storage and loss moduli of the alginate matrix, and $[\eta]$ is a parameter that for rigid spherical inclusions takes the value $[\eta]=2.5[35]$.
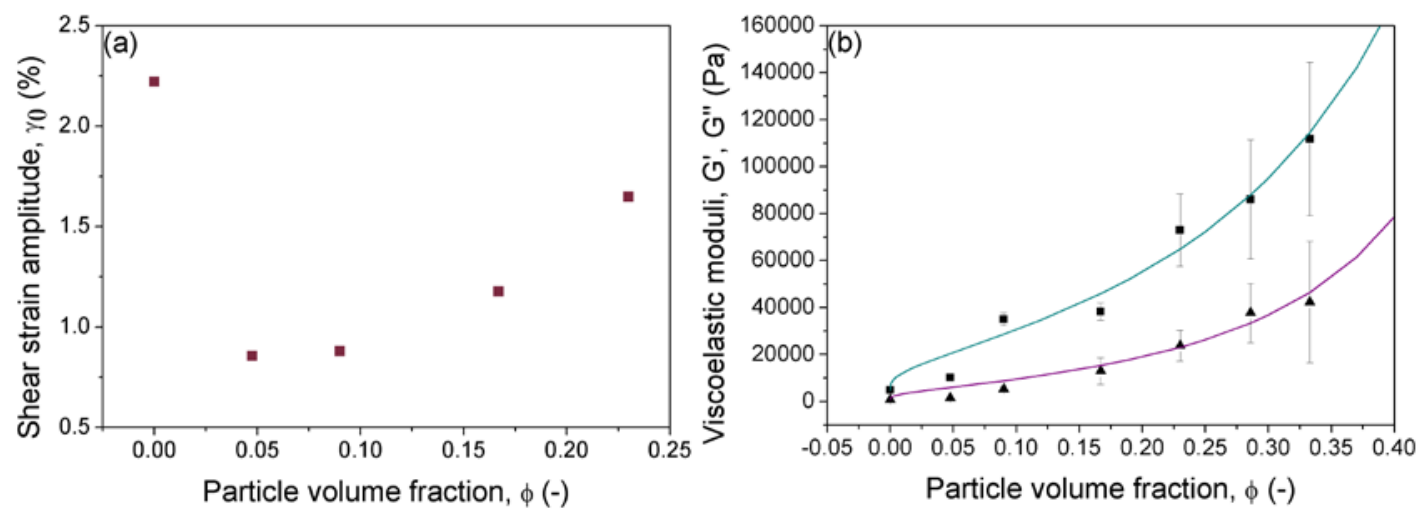

Figure 5. (a) Strain amplitude corresponding to the peak value in loss modulus $\left(G^{\prime \prime}\right)$; (b) Storage ( $\left.\boldsymbol{(}\right)$ and loss $(\boldsymbol{\Delta})$ moduli as functions of the volume fraction of iron particles in the magnetic hydrogels -values represent the average of $G^{\prime}$ and $G$ ' corresponding to the LVR, as determined by amplitude sweep tests. The continuous lines represent the best fits to equation (2). 
The validity of this expression has been previously demonstrated for magnetic hydrogels. For example, for magnetic hydrogels consisting of carrageenan polymer and carbonyl iron microparticles, Mitsumata et al. (2012) reported a good agreement between the prediction of $\mathrm{KD}$ equation and the experimental increase of the storage modulus with particle content up to a volume fraction of 0.23 [24]. On the contrary, we recently demonstrated that the enhancement of the mechanical properties with particle content largely exceeded such predictions in the case of magnetic hydrogels based on magnetic nanoparticles and fibrin polymers [17]. For these hydrogels, we found that the nanoparticles served as nuclei for the cross-linking of the fibrin polymer network, increasing hugely the number of polymer strands ending at a single cross-linking point (functionality of the cross-linking).

For the magnetic hydrogels of the present work, there is not a good fit with KD equation with all physically relevant values of the parameters $[\eta]$ and $\Phi_{m}$ and the discrepancy is higher at medium and high concentration of particles (fit not shown here). Similarly to the case of our previous work [17], we might be tempted to interpret this in terms of changes at the microscopic level in the polymer arrangement of the alginate network. However, alginate ions are negatively charged, whereas silica (coating of the surface of Fe-CC particles) is also negatively charged in water above $\mathrm{pH} 3$ [36]. Thus, electrostatic repulsion rather than electrostatic attraction is expected between the Fe-CC particles of the present work and the alginate strands. Nevertheless, weak hydrogen bonds between alginate macromolecules and silica-covered particles are expected even at opposite charges of both species [37]. These bonds just ensure some cohesion between the particles and the polymer strands. In fact, electron microscopy 
observations show the presence of polymer fibers attached to the surface of the particles, without changes in the polymer arrangement at the global scale (see Figure 3).

The question arising at this point is whether this cohesion between particles and alginate strands could positively contribute to the increase of the viscoelastic moduli of magnetic alginate hydrogels. To investigate this possibility, we also used bare (without silica coating) iron particles (Fe-HQ), for which alginate ions should show more affinity. As observed, for the same concentration of iron particles, the viscoelastic moduli exhibited by the magnetic hydrogels containing Fe-HQ particles were higher than these exhibited by the magnetic hydrogels containing Fe-CC particles (Figure 4b). Therefore, we can conclude that a stronger cohesion between particles and alginate strands (as in Fe-HQ sample) gives a stronger increase of the viscoelastic moduli. Note however that $\mathrm{KD}$ equation is based on non-slipping condition (i.e., strong cohesion) between particles and the polymer matrix, and consequently a stronger cohesion cannot result in experimental curves above the prediction of $\mathrm{KD}$ equation.

Another phenomenon that might have some influence on the measured values of the viscoelastic moduli is the existence of a gradient in concentration of magnetic particles within the hydrogel due to some gravitational settling during cross-linking. To investigate it, we measured the hydrogels containing iron particles turning up-and-down the samples prior to measurement. As observed, although the values of the viscoelastic moduli obtained for samples turned up-and-down were smaller than these of unturned samples, in general (and especially for the values of the storage modulus) there is a significant overlap of error bars of both data sets (Figure 4b). Therefore, even though 
the effect of a gradient in concentration of magnetic particles cannot be excluded, it does not seem to be relevant.

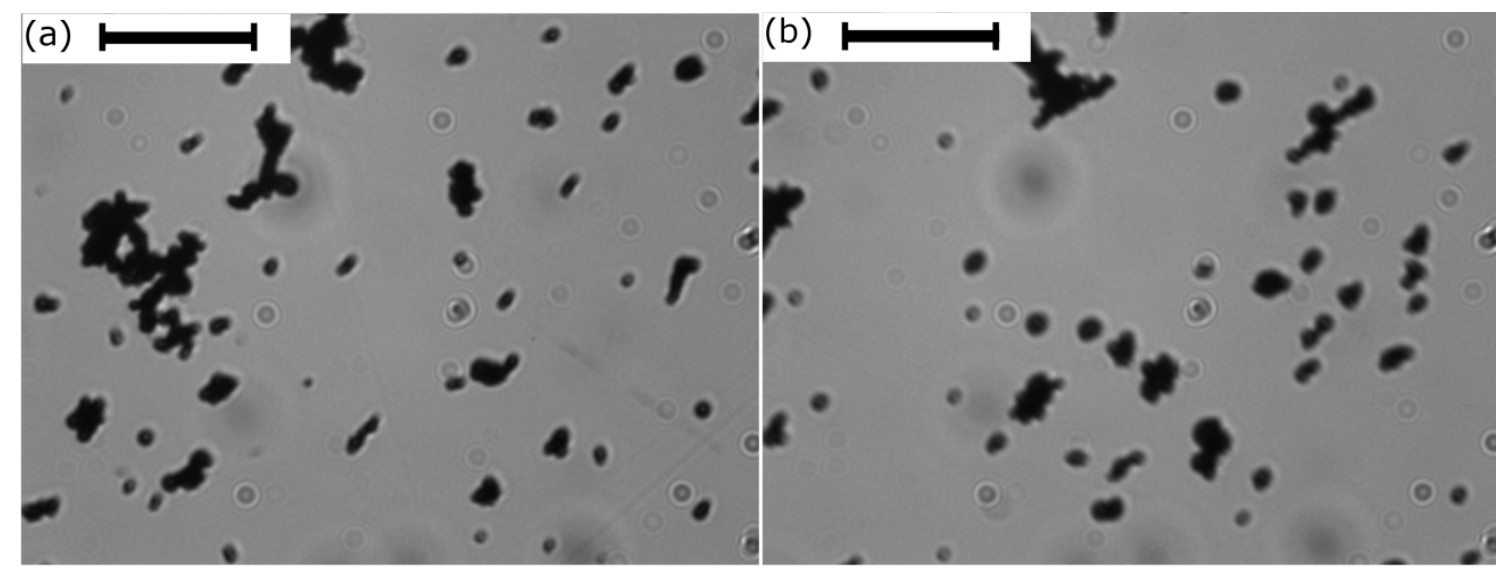

Figure 6. Optical microscopy images of diluted suspensions of Fe-CC particles in water: (a) and (b) represent images of different aliquots. Scale bar length 50 $\mu \mathrm{m}$.

Finally, discrepancies with respect to KD equation could be a consequence of the existence of some kind of aggregation between particles, which could form clusters appearing because of colloidal interactions and/or remnant magnetization of particles. The formation of clusters was in fact corroborated by SEM micrographs of the hydrogels (Figures 3b-d) and by observations by optical microscopy of diluted suspensions of iron particles in water (Figure 6). The existence of particle clusters would give rise to a higher effective concentration of rigid inclusions within the hydrogels than this of the true solid content -note that volume of a cluster is higher than the total volume of the particles constituting it. This hypothesis would justify the underestimation of the $\mathrm{KD}$ equation with respect to the experimental results. To account for this effect, we found that the following empirical equation (that still has some 
mathematical similarity with $\mathrm{KD}$ equation) fitted reasonably well our experimental concentration dependencies:

$$
G^{\prime}=G_{0}^{\prime}\left(1-\alpha^{\prime} \times \Phi^{n^{\prime}}\right)^{-\beta^{\prime}} ; G^{\prime \prime}=G^{\prime \prime}{ }_{0}\left(1-\alpha^{\prime \prime} \times \Phi^{n^{\prime \prime}}\right)^{-\beta^{\prime \prime}}
$$

where $\beta^{\prime}, \beta^{\prime}, \alpha^{\prime}, \alpha^{\prime}$ ' and $n^{\prime}, n^{\prime}$ are adjustable parameters, whose values are $\beta^{\prime}=\beta^{\prime \prime}=1.6, \quad \alpha=\alpha^{\prime \prime}=1.06 \pm 0.02, n^{\prime}=0.19 \pm 0.03$ and $n^{\prime \prime}=0.13 \pm 0.03$. As inferred from Figure 5b, Equation (2) provided a reasonably good fit of the concentration dependencies of the shear moduli in the range of particle volume fractions $0.05-0.33$. However, this equation does not reproduce a linear concentration dependency expected at lower concentrations, while extrapolation to higher concentrations does not have sense because of impossibility to prepare and handle the hydrogels above a particle volume fraction of approx. 0.35 .

We also analyzed the dependence of the viscoelastic moduli ( $G^{\prime}$ and $G^{\prime \prime}$ ) as a function of frequency within the LVR. Some typical curves for nonmagnetic and magnetic hydrogels are shown in Figure 7 -similar results were obtained for other concentrations. As observed, both $G^{\prime}$ and $G^{\prime \prime}$ increase slightly with the frequency of oscillation for the range of frequencies under study. Furthermore, in all cases $G$ ' was considerably larger than G', These tendencies are typical of cross-linked polymer systems [38]. In addition, results of Figure 7 corroborate the same tendencies and conclusions extracted above from amplitude sweep measurements (Figure 4) for the effects of modification of particle surface (Fe-HQ particles vs. Fe-CC particles) and existence of particle gradients. 


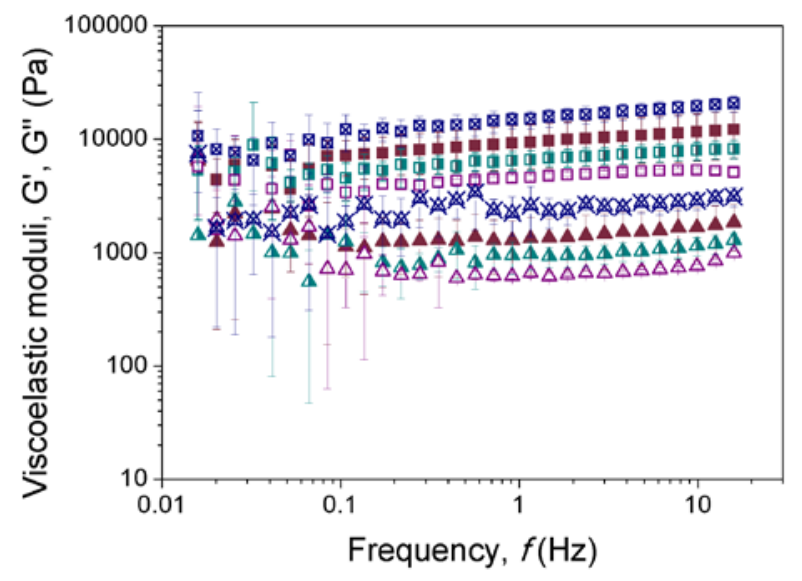

Figure 7. Storage (squares) and loss (triangles) moduli for nonmagnetic hydrogels and magnetic hydrogels (0.046 volume fraction of iron particles) as a function of the frequency of oscillatory measurements of fixed amplitude (0.03\%) within the LVR. $\square$ : nonmagnetic hydrogels; $\mathbf{n}:$ magnetic hydrogels containing Fe-CC particles without turning; a: magnetic hydrogels containing Fe-CC particles after turning up-and-down. $\otimes$ : magnetic hydrogels containing Fe-HQ particles.

\subsubsection{Rheological characterization in the presence of an applied magnetic field}

We analyzed the dependence of the storage $\left(G^{\prime}\right)$ and loss $\left(G^{\prime \prime}\right)$ moduli of magnetic hydrogels as a function of the strain amplitude in oscillatory measurements at fixed frequency of $1 \mathrm{~Hz}$, under application of magnetic fields of different strength. Similarly to the case in the absence of field, the experimental curves showed a typical trend of a viscoelastic solid-like material, characterized by an initial plateau-like region, which is identified as the LVR, and a sharp decrease of both moduli at large strain amplitude (Figure 8). From the analysis of Figure 8, we can conclude that the application of an 
external magnetic field resulted in an enhancement of the viscoelastic moduli, which became higher when the intensity of the magnetic field increased. This phenomenon is known as magnetorheological (MR) effect [39]. The enhancement of the viscoelastic moduli with the intensity of the applied magnetic field was also confirmed by measurements of the viscoelastic moduli as a function of frequency (frequency sweeps, not shown here). Besides, results of frequency sweeps under a magnetic field demonstrated similar trends of $G^{\prime}$ and $G^{\prime \prime}$ ' with frequency to those obtained in the absence of an applied magnetic field (Figure 7). Furthermore, as observed by comparison of curves in parts (a) and (b) of Figure 8, the storage modulus was much higher than the loss modulus for all the intensities of the applied field.
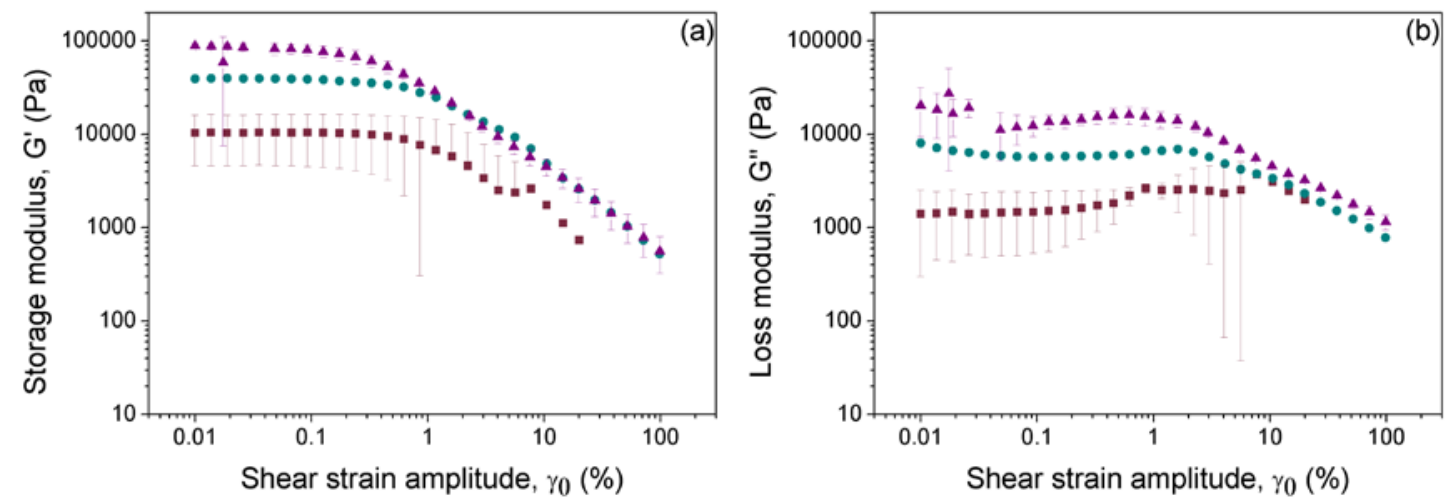

Figure 8. (a) Storage modulus and (b) loss modulus of magnetic hydrogels containing 0.046 volume fraction of magnetic particles (Fe-CC), as functions of the strain amplitude for oscillatory measurements at a fixed frequency of 1 Hz. Magnetic field strength, $H: \| 0 \mathrm{kA} / \mathrm{m}, \bullet 156 \mathrm{kA} / \mathrm{m}$ and $\boldsymbol{\Delta} 282 \mathrm{kA} / \mathrm{m}$. Note that the same axis scale is used in both parts.

In order to analyze the MR effect as a function of the concentration of particles, we plotted the average values of $G^{\prime}$ corresponding to the LVR as functions of the concentration of magnetic particles both in the absence of 
magnetic field and in the presence of the strongest magnetic field under study, $H=282 \mathrm{kA} / \mathrm{m}$ (Figure 9). As observed, the larger the concentration of magnetic particles, the higher the resulting enhancement of $G^{\prime}$ under application of a magnetic field. Obviously, this enhancement must be related with the magnetic character of the Fe-CC particles, which get magnetized and attract each other under the application of a magnetic field -note that these particles are multidomain from the magnetic point of view. MR effect in liquid media (i.e., MR fluids) is characterized by magnetic field-induced aggregation of the magnetizable particles, giving rise to particle column-like structures aligned in the direction of the magnetic field. These particle structures oppose to the deformation induced by the shear forces, resulting in enhanced rheological moduli under a field [39].

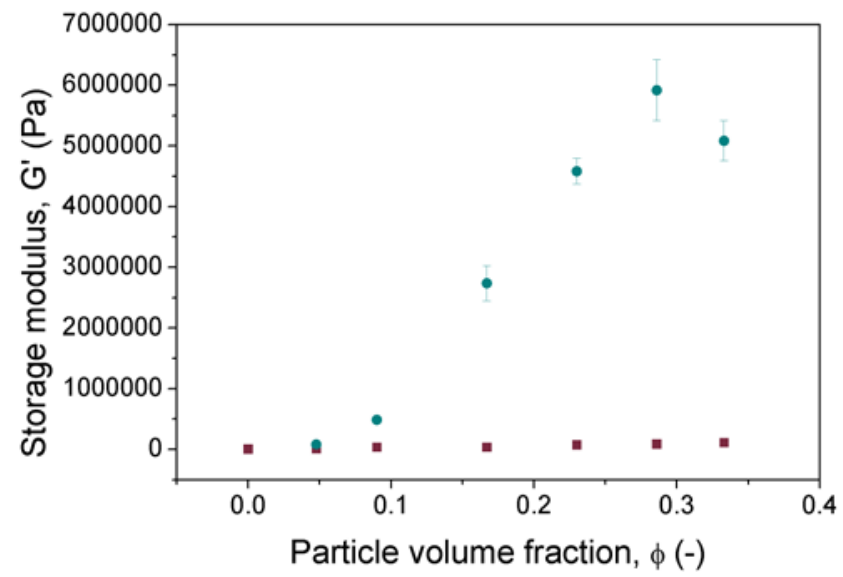

Figure 9. Storage modulus ( $\left.G^{\prime}\right)$ corresponding to the LVR as a function of the concentration of Fe-CC particles in the magnetic hydrogels. Values represent the average of $G^{\prime}$ corresponding to the LVR, as determined by amplitude sweep tests. Magnetic field strength, $H$ : $0 \mathrm{kA} / \mathrm{m}, \bullet 282 \mathrm{kA} / \mathrm{m}$. Here, the storage modulus for nonmagnetic hydrogels is $G^{\prime}=4800 \pm 200 \mathrm{~Pa}$. 
In the present case of the viscoelastic alginate matrix, formation of columnlike structures is expected to be partially hindered by the matrix elasticity. However, the matrix cannot completely avoid the relative motion of neighboring magnetic particles. Since the hydrogel storage modulus in the presence of field is much higher than in the absence of field (Figures 8a and 9), magnetic interactions between particles or clusters are expected to be quite strong as compared to elastic forces and the particles are expected to displace towards each other at distances a few times higher than those dictated by the global applied strain in the absence of field. If the local stress generated by the particle displacement is below the hydrogel yield stress, the particle displacement should be reversible with respect to the magnetic field application, and the particle space distribution under applied shear and magnetic fields is expected to be governed by the minimum of the sum of the elastic and magnetic parts of the hydrogel free energy [40], while the hydrogel moduli- by the energy change with respect to the strain. It is therefore clear that increasing magnetic field will provide stronger local displacement of particles, which will result in stronger anisotropy of field-induced spatial distribution of particles and, along with increasing magnetic forces between particles, this will increase the hydrogel elastic moduli, as observed in Figure 10. At the same time, increasing particle concentration means shortening the average distances between magnetic particles and thus enhancement of magnetic interactions between them. Since at relatively large particle displacements (that might occur thanks to magnetic interactions even at low global strains) the local elastic moduli decrease with local strain, we might expect a quite strong increase of 
the local particle displacements with increasing particle concentrations and fields that can explain the two following observed effects: (a) a stronger concentration dependence of shear moduli in the presence of field (Figure 9) as compared to relatively weak slightly non-linear concentration dependence of the MR effect in MR fluids; (b) a decrease of the extension of the LVR (described by the critical strain that marks the onset of the non-linear regime) with increasing magnetic field (Figure 11a) caused by increase of local strains because of larger displacement of particles. At this point, it is worthy to mention that X-ray tomography has previously been used for the investigation of the microstructure formation in MR elastomers [41]. This technique might provide valuable information regarding the formation and evolution of particle aggregates under an applied magnetic field in magnetic hydrogels, and thus, it might be used for the investigation of competition between elastic and magnetic forces at the microscopic level. Future works in this sense might be relevant for the development of realistic microstructural models of the MR properties of magnetic hydrogels.

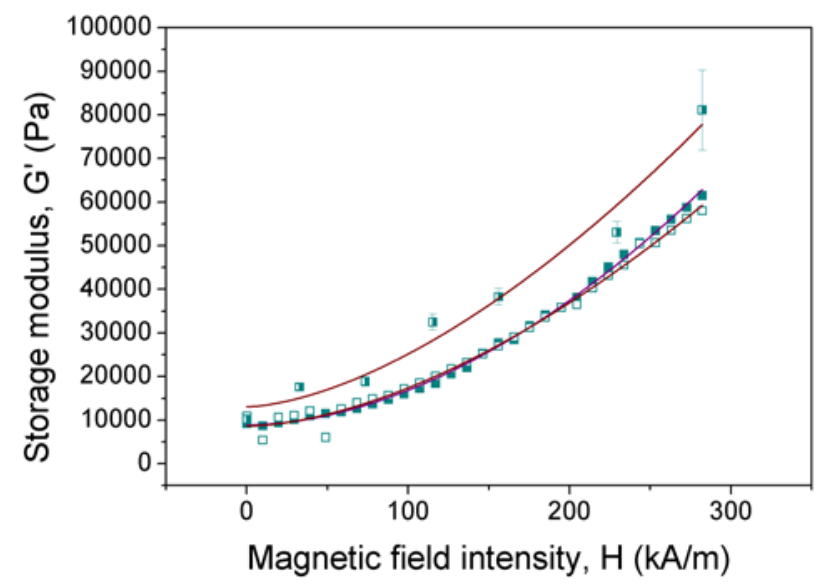


Figure 10. Storage modulus as a function of the applied magnetic field for magnetic hydrogels containing 0.046 volume fraction of magnetic particles. $\square$ : Values represent the average of $G$ ' corresponding to the LVR, as determined by amplitude sweep tests; note that a new sample was used for each value of the applied field. $\square, \square$ : Results for magnetic field sweep using one sample along the sweep -results for two different samples are shown; full symbols and open symbols represent different samples. Lines represent best fits to equation (3).

Let us now analyze in more details the influence of the magnetic field on the storage modulus. For this aim we characterized the rheological behavior of the magnetic hydrogels containing 0.046 volume fraction of magnetic particles under magnetic fields of different intensity (Figure 10). We performed the analysis in two different ways. Firstly, by using a new sample for each value of the applied magnetic field and subjecting it to an amplitude sweep test under the selected value of magnetic field (Figure 10). Secondly, by subjecting a given sample to a sweep of magnetic field strength, starting at zero field (Figure 10). In both cases we obtained a stronger than linear enhancement of the storage modulus with the magnetic field strength. Note also that the MR effect obtained by using a new sample for each value of the magnetic field was slightly higher than this obtained by sweeping the field for a given sample. This difference might be connected to the differences in the protocols (i.e., sample's histories) used for fresh samples and samples subjected to magnetic sweep tests (see subsection 2.3). 
Various theoretical approaches are suggested in literature for the description of the rheological properties of magnetic gels -see for example Ref. [42] and the overviews in Refs. [13-14]. However, the model in Ref. [42] deals with the case when the particles cross-link the gel network and, therefore, the particle size is smaller than the typical size of the net cell. In contrast, in the case of the present magnetic alginate hydrogels, the size of the particles is much larger than that of the net cell, and thus the gel can be considered as a continuous medium with respect to the particles. Our analysis shows that none of the other microscopic models reported in literature (see Refs. $[13,14]$ ) can describe the experimental results shown in Figure 10. Because of this reason, in this manuscript we focus only on the phenomenological description of the MR effect exhibited by magnetic alginate hydrogels.

From a qualitative viewpoint, the observed strong MR effect can be explained by unification of the particle clusters (evidenced in Figure 6), into chain-like, column-like or other heterogeneous structures, under the action of the applied magnetic field. A similar field-induced particle aggregation takes place in MR fluids, for which various theoretical and experimental studies demonstrate power-law dependencies of relevant rheological parameters (e.g., storage modulus) on the magnetic field strength, $H$ [23, 43]. Based on this consideration, we used the following equation to fit the experimental results of G' shown in Figure 10:

$$
G^{\prime}=\delta \times H^{p}+G_{0}^{\prime}
$$

Here $G^{\prime}{ }_{0}$ is the storage modulus in the absence of an applied magnetic field, and $\delta, p$ are fitting parameters. As observed in Figure 10, equation (3) fits well to the experimental results. The best-fit values for the exponent are $p=1.6 \pm 0.2$ for the curve representing 
the average of $G^{\prime}$ corresponding to the LVR, and $p=1.84 \pm 0.03, p=1.69 \pm 0.07$ for the curves representing magnetic sweep tests. These exponents lie in the range $1 \leq p \leq 2$, whose lower bound $(p=1)$ was predicted by the model in Ref. [43] of affine displacement of particles with non-linear magnetization in a linear chain and whose upper bound $(p=2)$ is predicted by a point dipole approximation applied to low magnetic fields [23].
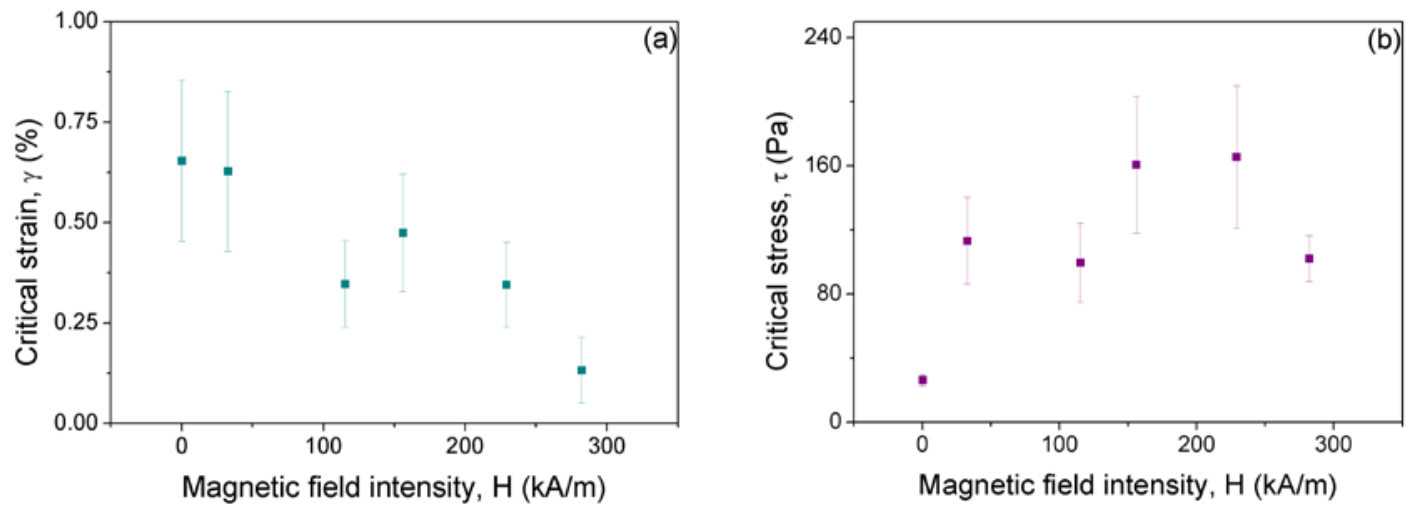

Figure 11. (a) Critical strain amplitude and (b) critical stress amplitude that mark the onset of the nonlinear viscoelastic regime as a function of the intensity of the applied magnetic field for magnetic hydrogels containing 0.046 volume fraction of Fe-CC particles.

Other rheological parameters may also depend on the intensity of the applied magnetic field. It is the case of the critical strain amplitude and critical stress amplitude that mark the onset of the nonlinear viscoelastic regime. Using the same criterion as in subsection 3.2.1 for the limit of the LVR, we obtained that the critical strain decreased as the magnetic field was increased (Figure 11a). As for the critical stress, it was considerably higher under an applied magnetic field than in its absence, although there were not significant differences for the different intensities of the applied field (Figure 11b). These results for the critical strain and stress in combination with the previously 
discussed enhancement of the storage modulus indicate that the magnetic hydrogels became stronger, but more fragile, as the magnetic field strength increased. The increase in fragility was likely due to the particles undergoing a stronger relative displacement under an applied magnetic field, which should lead to higher local strains and thus higher fragility.

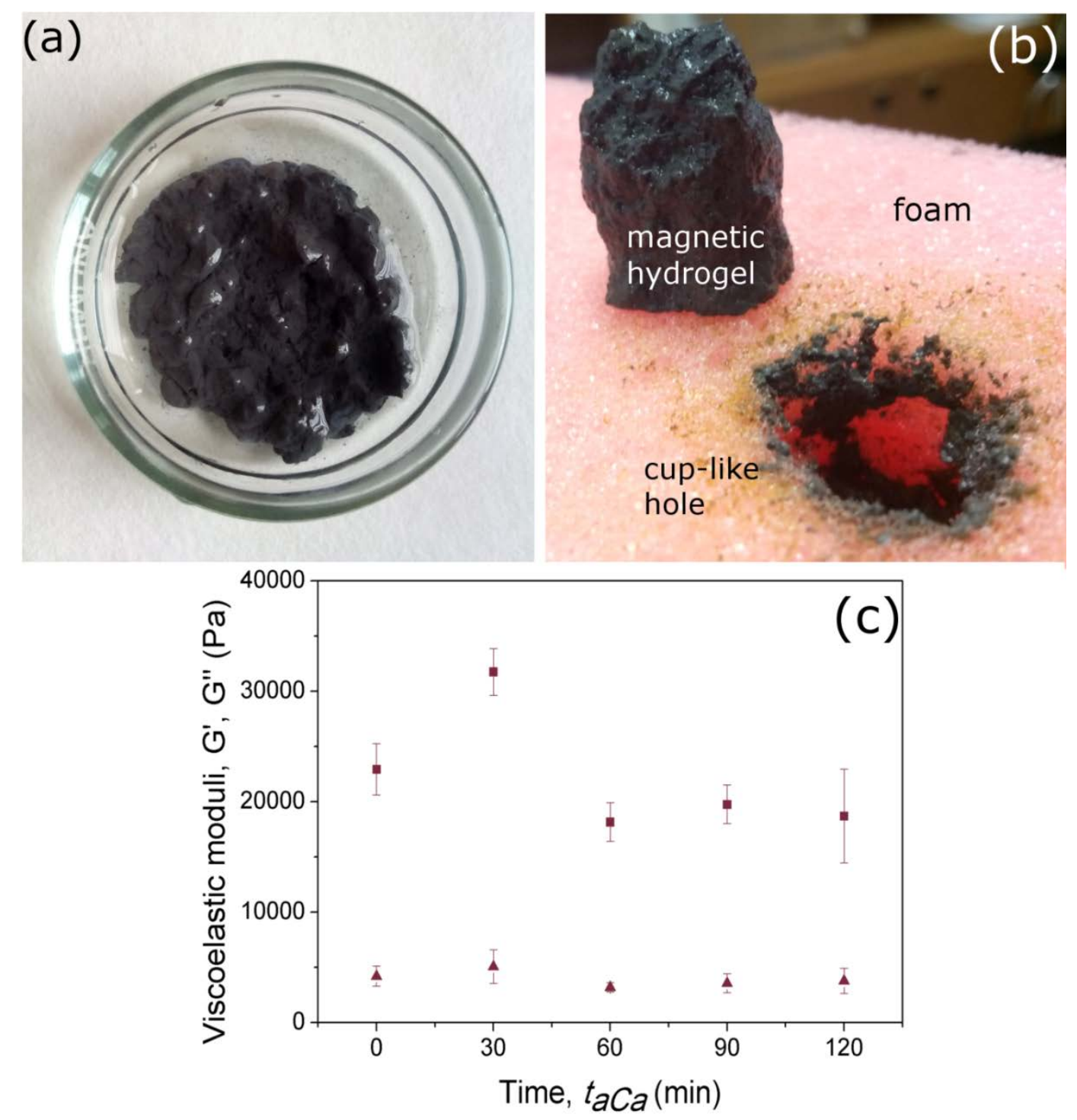

Figure 12. (a-b) Injected magnetic hydrogel after 24 hours of $\mathrm{CaCl}_{2}$ addition. Picture (a) corresponds to a magnetic hydrogel injected in a Petri dish. Picture (b) corresponds to a magnetic hydrogel injected through foam (also shown) in 
a previously pierced cup-like hole -note that there was not diffusion of the hydrogel to surrounding areas. Time $\left(t_{\mathrm{aCa}}\right)$ of addition of the $\mathrm{CaCl}_{2}$ solution: 30 minutes -a similar aspect was obtained for different values of $t_{\mathrm{aCa}}$. (c) Storage $(\boldsymbol{\square})$ and loss $(\boldsymbol{\Delta})$ moduli, representing the average corresponding to the LVR, of magnetic hydrogels as a function of $t_{\mathrm{aCa}}$. Volume fraction of magnetic particles was 0.046 in all cases.

\subsection{Injectability study}

As described in subsection 2.4, we recovered the injected magnetic hydrogels after 24 hours of the addition of the $\mathrm{CaCl}_{2}$ solution, and immediately afterwards we analyzed their macroscopic integrity and rheological properties. It should be noted that although the resulting hydrogels presented a lumpy appearance, each of them consisted of one solid-like piece that could be manipulated without fracture (Figure 12a-b). Concerning their rheological properties, we obtained the storage modulus ( $\left.G^{\prime}\right)$ and loss modulus $\left(G\right.$ ’') corresponding to the LVR and plotted them as a function of the time $\left(t_{\mathrm{aCa}}\right)$ elapsed from the moment of addition of the $\mathrm{CaCl}_{2}$ solution (Figure 12c).

As observed, for the whole range of $t_{\mathrm{aCa}}$ the resulting hydrogels presented a solidlike viscoelastic behavior, characterized by $G^{\prime}$ values larger than $G^{\prime \prime}$ values. Concerning the role of $t_{\mathrm{aCa}}$, it was negligible on the magnitude of $G$ ', whereas in the case of $G^{\prime}$ a maximum value was obtained for $t_{\mathrm{aCa}}=30 \mathrm{~min}$. Furthermore, in all cases the values of $G^{\prime}$ and $G$ ', were of the same order of magnitude than those reported in subsection 3.2.1 for magnetic hydrogels not subjected to injection -e.g., $G^{\prime}=10200 \pm$ $400 \mathrm{~Pa}$ for the hydrogel not subjected to injection and an average of $G^{\prime}=22000 \pm 6000$ Pa for the injected hydrogels. Therefore, the defined protocol of injection demonstrated 
to be adequate for retaining the morphology and mechanical characteristics of magnetic alginate hydrogels and might be adequate for implementation in vivo. At this point note that ideal injectable medical hydrogels should meet several general requirements including biocompability and biodegradability, as well as ease injection through a syringe to alleviate the pain of the patient, and rapid gelation after injection to avoid diffusion to surrounding areas [44-45]. Furthermore, for certain applications other specific requirements are needed, such as similar mechanical properties to target tissues in tissue engineering applications.

\section{Conclusions}

We have reported a comprehensive analysis of the structure and mechanical properties of magnetic alginate hydrogels, consisting of micronsized iron particles embedded within an ionic alginate polymer network. We have designed a two-step protocol that allows obtaining macroscopically homogeneous magnetic alginate hydrogels containing as much as 0.33 volume fraction of iron microparticles. However, from the microscopic analysis we have found that iron microparticles are aggregated into clusters with number of particles per aggregate being of the order of ten. These clusters are nevertheless homogeneously dispersed within the polymer network.

From the rheological analysis under shear in the absence of applied magnetic field we have found that the hydrogels, both nonmagnetic and magnetic, presented a typical behavior of a cross-linked polymer system, characterized by values of the storage modulus higher than these of the loss modulus within the linear viscoelastic region. What is more, our experimental results have demonstrated an intense enhancement of the viscoelastic moduli with the concentration of magnetic particles. At a first glance, this enhancement seems stronger than this expected for composites of hard spheres 
within a polymer matrix according to classical laws, such as Krieger-Dougherty (KD) law. However, based on experimental evidences, we have proven that the strong concentration behavior does not come from changes in the polymer network induced by inclusion of particles, as it was the case in our previous study with fibrin-based hydrogels [17]. In the present study, the particle concentration dependence of the viscoelastic moduli is likely due to the formation of particle clusters, whose volume is larger than the total volume of the particles. We have found that an empirical equation that has some mathematical similarity with the $\mathrm{KD}$ equation fits well to the experimental dependence of the viscoelastic moduli on the particle concentration.

The analysis of the rheological behavior under applied magnetic fields has shown an intense strengthening of the magnetic hydrogels (increase of viscoelastic moduli) with the intensity of the magnetic field, especially at high volume fraction of magnetic particles (higher than 0.15). We have demonstrated that this strengthening follows a power law with the magnetic field, with exponent $p$ in the range $1<p<2$, corresponding to the predications of the Ginder's model [43] (valid for intermediate magnetic fields) and of the point dipole approximation [23] (valid at low magnetic fields). In the case of the magnetic hydrogels of the present work, the strengthening of the hydrogels with the magnetic field (i.e., the MR effect) is expected to be due to the appearance of strong magnetic forces between neighboring particle clusters. The relatively strong concentration dependence of the MR effect is explained by the synergy of the two following effects: (a) appearance, at the stage of the synthesis of the magnetic hydrogels, of dense particle clusters that under a magnetic field interact more strongly than separate particles; (b) increase of the local cluster displacements within the alginate matrix (resulting in stronger interactions) with increasing particle concentrations. 
Finally, we have proposed a protocol for the injection of magnetic alginate hydrogels. The starting point is a partially dehydrated magnetic hydrogel that can be injected using a syringe. The subsequent injection of a solution of $\mathrm{CaCl}_{2}$ gives rise to a macroscopically homogeneous magnetic hydrogel that presents viscoelastic moduli of the same order of magnitude that magnetic hydrogels prepared by the two-step protocol reported in the present work.

To conclude, we have reported protocols for the preparation and injection of homogeneous magnetic hydrogels, and extensively explored their versatile mechanical properties. From the fundamental viewpoint, future work will be required to construct microstructural models that describe accurately the MR effects developed by soft magnetic hydrogels like these studied in the present manuscript. From another perspective, the results of our work should constitute a reference for authors working on technological or biomedical applications of magnetic hydrogels. Future studies with laboratory animals are needed to fully evaluate the potential of our two-step protocol to be implemented in vivo.

\section{Acknowledgements}

This study was supported by projects FIS2013-41821-R (Plan Nacional de Investigación Científica, Desarrollo e Innovación Tecnológica, MINECO, Spain, cofunded by ERDF, European Union) and FIS2017-85954-R (Agencia Estatal de Investigación, AEI, Spain, co-funded by Fondo Europeo de Desarrollo Regional, ERDF, European Union). AZ is grateful to the program of the Ministry of Education and Science of the Russian Federation, projects 02.A03.21.0006; 3.1438.2017/4.6; 3.5214.2017/6.7 as well as to the Russian Fund of Basic Researches, project 18-08- 
00178. PK acknowledges financial support of the French government, piloted by the National Research Agency (ANR) in the framework of the project Future Investments UCAJEDI, Ref. No. ANR-15-IDEX-01 (RheoGels).

\section{References}

[1] Caló, E., and V. V. Khutoryanskiy,“Biomedical applications of hydrogels: A review of patents and commercial products,” European Polymer Journal 65, 252267(2015).

[2] Langer, R., “New methods of drug delivery,” Science 249, 1527-1533 (1990).

[3] Mitragotri, S., and J. Lahann, “Physical approaches to biomaterial design,” Nature Materials 8, 15-21 (2009).

[4] Choi, N.W., M. Cabodi,B. Held,L. Gleghorn,,J. Bonassar, and A. D. Stroock,“Microfluidific scaffolds for tissue engineering,” Nature Materials 6, 908915 (2007).

[5] Atala, A., S. B. Bauer, S. Soker, J. J. Yoo, and A. B. Retik,“Tissue-engineered autologous bladders for patients needing cystoplasty,” Lancet367, 1241-1246 (2006).

[6] Boucard, N.,C. Viton, D. Agay, E. Mari, T. Roger, Y. Chancerelle, and A. Domard, "The use of physical hydrogels of chitosan for skin regeneration following thirddegree burns,” Biomaterials 28, 3478-3488 (2007). 
[7] Yang, H., S. B. Hong, C. W. Park, K. Lee, Y. S. Choi, J. Yu, B. Seo, and J. Moon, “Magnetic Adsorbents Embedded in Hydrogel Bead for Surface Decontamination,” Journal of Nanoscience and Nanotechnology 16, 8569-8574 (2016).

[8] Hua, R., and Z. Li, "Sulfhydryl functionalized hydrogel with magnetism: Synthesis, characterization, and adsorption behavior study for heavy metal removal," Chemical Engineering Journal249, 189-200 (2014).

[9] Koetting, M. C., J. T. Peters, S. D. Steichen, and N. A. Peppas, "Stimulusresponsive hydrogels: Theory, modern advances, and applications,” Materials Science and Engineering: R: Reports 93, 1-49 (2015).

[10]Culver, H. R., J. R. Clegg, and N. A. Peppas, “Analyte-Responsive Hydrogels: Intelligent Materials for Biosensing and Drug Delivery,” Acc. Chem. Res.50, 170_ 178 (2017).

[11]Luchini, A., D. H. Geho, B. Bishop, D. Tran, C. Xia, R. L. Dufour, C. D. Jones, V. Espina, A. Patanarut, W. Zhou, M. M. Ross, A. Tessitore, E. F. Petricoin, and L. A. Liotta, "Smart Hydrogel Particles: Biomarker Harvesting: One-Step Affinity Purification, Size Exclusion, and Protection against Degradation,” Nano Lett. 8, 350-361 (2008).

[12]Yan, L., Z. Zhu, Y. Zou, Y. Huang, D. Liu, S. Jia, D. Xu, M. Wu, Y. Zhou, S. Zhou, and C. J. Yang, “Target-Responsive "Sweet” Hydrogel with Glucometer Readout for Portable and Quantitative Detection of Non-Glucose Targets,” J. Am. Chem. Soc.135, 3748-3751 (2013). 
[13]Lopez-Lopez, M. T., J. D. G. Durán, L. Y. Iskakova, and A. Y. Zubarev, “Mechanics of Magnetopolymer Composites: A Review,” Journal of Nanofluids5, 479-495 (2016).

[14]Weeber, R., M. Hermes, A. M. Schmidt and C. Holm, "Polymer architecture of magnetic gels: a review”, J. Phys.: Condens. Matter 30, 063002 (2018).

[15]Messing, R., N. Frickel, L. Belkoura, R. Strey, H. Rahn, S. Odenbach, and A. M. Schmidt, "Cobalt Ferrite Nanoparticles as Multifunctional Cross-Linkers in PAAmFerrohydrogels,” Macromolecules 44, 2990-2999 (2011).

[16]Ilg, P., “Stimuli-responsive hydrogels cross-linked by magnetic nanoparticles,”Soft Matter 9, 3465-3468 (2013).

[17]Bonhome-Espinosa, A. B., F. Campos, I. A. Rodriguez, V. Carriel, J. A. Marins, A. Zubarev, J. D. G. Duran, and M. T. Lopez-Lopez, “Effect of particle concentration on the microstructural and macromechanical properties of biocompatible magnetic hydrogels,” Soft Matter 13, 2928-2941, (2017).

[18]López-López, M. T., G. Scionti, A. C. Oliveira, J. D. G. Durán, A. Campos, M. Alaminos, and I. A. Rodríguez, "Generation and Characterization of Novel Magnetic Field-Responsive Biomaterials,” PLoS ONE 10 (2015).

[19]Wu, J., X. Gong, Y. Fan, and H. Xia, “Physically crosslinked poly(vinyl alcohol) hydrogels with magnetic field controlled modulus,” Soft Matter 7, 6205-6212 (2011). 
[20]Ramanujan, R.V., and L. L. Lao, “The mechanical behavior of smart magnethydrogel composites,” Smart Mater. Struct. 15, 952-956 (2006).

[21]Zhao, X.,J. Kim, C. A. Cezar, N. Huebsch, K. Lee, K. Bouhadir, and D. J. Mooney, “Active scaffolds for on-demand drug and cell delivery,” PNAS 108, 67-72 (2011).

[22]Nair, L.S., Injectable Hydrogels for Regenerative Engineering (Imperial College Press, United States, 2016).

[23]Bossis, G., O. Volkova, S. Lacis, and A. Meunier, "Magnetorheology: Fluids, Structures and Rheology,” LNP 594, 202-230 (2002).

[24] Mitsumata, T., A. Honda, H. Kanazawa, and M. Kawai, "Magnetically tunable elasticity for magnetic hydrogels consisting of carrageenan and carbonyl iron particles,” J. Phys. Chem. B 116, 12341-12348 (2012).

[25]Lee, K. Y., and D. J. Mooney, “Alginate: Properties and biomedical applications,” Progress in Polymer Science 37, 106-126 (2012).

[26]Utech, S., R. Prodanovic, A. S. Mao, R. Ostafe, D. J. Mooney, and D. A. Weitz, "Microfluidic generation of monodisperse, structurally homogeneous alginate microgels for cell encapsulation and 3D cell culture,” Adv. Healthcare Mater. 4, 1628-1633 (2015).

[27]Tan, W.,and S. Takeuchi,"Monodisperse Alginate Hydrogel Microbeads for Cell Encapsulation,” Adv. Mater. 19, 2696-2701 (2007). 
[28] Mredha, T. I., Y. Z. Guo, T. Nonoyama, T. Nakajima, T. Kurokawa, and J. P. Gong, “A Facile Method to Fabricate Anisotropic Hydrogels with Perfectly Aligned Hierarchical Fibrous Structures,” Adv. Mater. 30, 1704937 (2018).

[29] Moghimi, E., A. R. Jacob, N. Koumakis, and G. Petekidis, “Colloidal gels tuned by oscillatory shear,” Soft Matter 13, 2371-2383 (2017).

[30] Aguirre-Olabide, I., M. J. Elejabarrieta, and M. M. Bou-Ali, “Matrix dependence of the linear viscoelastic region in magnetorheological elastomers," Journal of Intelligent Material Systems and Structures 26, 1880-1886 (2015).

[31]Wereley, N. M., A. Chaudhuri, J. H. Yoo, S. John, S.Kotha, A. Suggs, R. Radhakrishnan, B. J. Love, and T. S. Sudarshan, "Bidispersemagnetorheological fluids using Fe particles at nanometer and micron scale,” Journal of Intelligent Material Systems and Structures 17, 393-401 (2006).

[32]Christensen, R. M., Mechanics of Composite Materials (Wiley, New York, 1979).

[33] Batchelor, G.K., and J. T. Green, "The determination of the bulk stress in a suspension of spherical particles to order c²,” J. Fluid Mech. 56, 401-427 (1972).

[34]Krieger, I.M., and T. J. Dougherty, “A mechanism for non-Newtonian flow in suspension of rigid spheres,” Trans. Soc. Rheol. 3, 137-152 (1959).

[35]Larson, R.G., The Structure and Rheology of Complex Fluids (Oxford, New York, 1999). 
[36] Mandel, K., M. Straßer, T. Granath, S. Dembski, and G. Sextl, "Surfactant free superparamagnetic iron oxide nanoparticles for stable ferrofluids in physiological solutions,” Chem. Commun. 51, 2863-2866 (2015).

[37]Mittal, V., Nanocomposites with Biodegradable Polymers: Synthesis, Properties, and Future Perspectives (Oxford University Press, New York, 2011).

[38] Macosko, C.W., Rheology: Principles, Measurements, and Applications (WileyVCH, 1994).

[39]Bossis, G., S. Lacis, A. Meunier, and O. Volkova, "Magnetorheological fluids," Journal of Magnetism and Magnetic Materials 252, 224-228 (2002).

[40]Biller, A. M., O. V. Stolbov, and Y. L. Raikher, "Modeling of particle interactions in magnetorheological elastomers,” J. Appl. Phys. 116, 114904 (2014).

[41]Borbáth, T., S. Günther, D.Yu. Borin, Th. Gundermann, and S. Odenbach, "X $\mu C T$ analysis of magnetic field-induced phase transitions in magnetorheological elastomers,” Smart Mater. Struct. 21, 105018 (2012).

[42] Pessot, G., H. Löwen, and A. M. Menzel, "Dynamic elastic moduli in magnetic gels: Normal modes and linear response,” J. Chem. Phys. 145, 104904 (2016).

[43] Ginder, J. M., L.C. Davis, and L.D. Elie, "Rheology of magnetorheological fluids: models and measurements,” Int. J. Mod. Phys B. 10, 3293 (1996).

[44]Yu, L., and J. Dign, “Injectable hydrogels as unique biomedical materials,” Chem. Soc. Rev. 37, 1473-1481 (2008). 
[45] Yan, S., T. Wang, X. Li, Y. Jian, K. Zhang, G. Li, and J. Yin, "Fabrication of injectable hydrogels based on poly(Lglutamicacid) and chitosan,” RSC Adv. 7, 17005-17019 (2017). 